\title{
A systematic uncertainty on the energy scale of the Telescope Array fluorescence detectors
}

\author{
Toshihiro Fujii $^{*, a}$, Masaki Fukushima ${ }^{a}$, Daisuke Ikeda ${ }^{a}$, Dmitri Ivanov ${ }^{b}$, Jon Paul \\ Lundquist $^{b}$, Bokkyun Shin ${ }^{c}$, Gordon B. Thomson ${ }^{b}$, Yoshiki Tsunesada ${ }^{c}$ for the \\ Telescope Array Collaboration ${ }^{\dagger}$ \\ ${ }^{a}$ Institute for Cosmic Ray Research, University of Tokyo, Kashiwa, Chiba, 277-8583 JAPAN \\ ${ }^{b}$ High Energy Astrophysics Institute and Department of Physics and Astronomy, University of \\ Utah, Salt Lake City, UT, USA \\ ${ }^{c}$ Graduate School of Science, Osaka City University, Osaka, Japan \\ E-mail:
}

The Telescope Array experiment (TA) is the largest cosmic-ray detector in the northern hemisphere and consists of a surface detector (SD) array, plus three fluorescence detector (FD) stations overlooking the SD. The large field-of-view of an FD allows for reconstruction of the air-shower development in the atmosphere by imaging ultra-violet fluorescence light from atmospheric nitrogen excited by UHECRs. In estimation of the primary energy it is necessary to add to the calorimetric energy observed by the FD a "missing energy", meaning the fraction of the primary energy that is not deposited by charged particles in the air. We report on the measurement of the missing energy from observed data collected by the TA FD and TA SD, independently of Monte Carlo simulations, using a technique pioneered by the Pierre Auger Observatory. We also address the effect on the energy scale attributed to fluorescence yield parameters.

35th International Cosmic Ray Conference - ICRC2017

10-20 July, 2017

Bexco, Busan, Korea

\footnotetext{
* Speaker.

${ }^{\dagger}$ Full author list: http://www.telescopearray.org/index.php/research/collaborators
} 


\section{Introduction}

The fluorescence detector (FD) is one of the well-established methods to observe an extensive air shower (EAS) induced by ultrahigh-energy cosmic rays (UHECRs). The large field-of-view of an FD allows for reconstruction of the air-shower longitudinal development in the atmosphere by imaging ultra-violet fluorescence light from atmospheric nitrogen excited by the EAS.

The Telescope Array Experiment (TA) is the largest cosmic-ray detector in the northern hemisphere [四]. TA consists of 507 surface detectors (SDs) deployed on a square grid with $1.2 \mathrm{~km} \mathrm{spac}-$ ing, covering an effective area of about $700 \mathrm{~km}^{2}$ []] , overlooked by 48 FDs at three locations [B]]. One FD station located northwest of the SD array, consists of 24 FDs including 10 with higher elevation angles, which were previously used in the High Resolution Fly's Eye (HiRes) experiment [四]. Two other stations at the array's southeast and southwest consist of 12 newly designed and constructed FDs [3] with new calibrations [ [5, 目, 四]. In order to calibrate the atmospheric conditions and relative gains among the three FD stations, a Central Laser Facility (CLF) is installed at the center of the TA site [8]]. An ultraviolet laser with a wavelength of $355 \mathrm{~nm}$ fires a 300 vertical shots every 30 minutes. The atmospheric transmittance was monitored at the start and end of daily operation of the FD by a Light Detection And Ranging (LIDAR) system by May 2012, which measured the back-scattered photons from an ultraviolet laser [Q]. The TA has an electron light source (ELS) to understand the detector characteristics and the fluorescence yield by an artificial EAS at a distance of $100 \mathrm{~m}$ from FD [Ш]]. Photographs of these FDs, LIDAR and ELS are shown in the Figure 田.

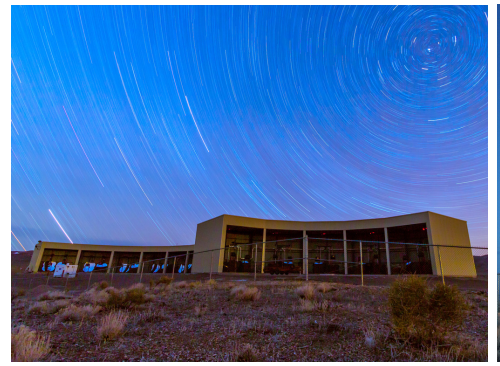

(a) HiRes refurbished FDs.

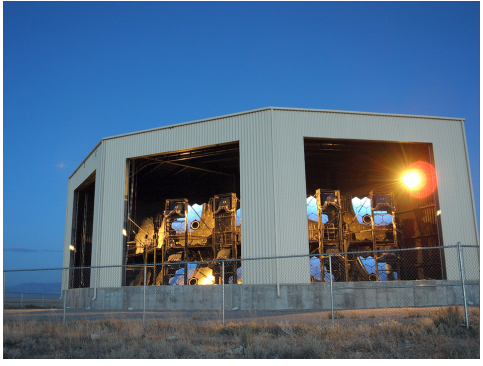

(b) Newly constructed FDs

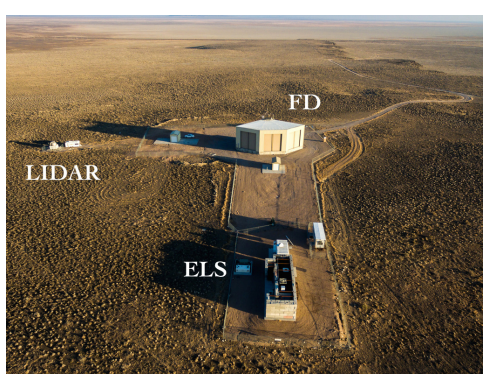

(c) FD station, LIDAR and ELS.

Figure 1: (a) HiRes refurbished FDs located northwest of the SD array. (b) Newly constructed FDs at the array's southeast and southwest. (c) The southeast FD station together with LIDAR and ELS.

\section{Primary energy determination with FD}

To infer the atmospheric energy deposit in the EAS from the light measured by the detector, we must specify both a fluorescence yield and an atmospheric model, which respectively describe the production and attenuation of light prior to detection. In estimation of the primary energy it is necessary to add to the calorimetric energy observed by the FD a "missing energy", meaning the fraction of the primary energy that is not deposited by charged particles in the air. Conventionally the missing energy is estimated by the Monte Carlo Simulation, e.g. CORSIKA [W]]. However, the Pierre Auger Observatory reported a method to evaluate the missing energy using the observables 
with a fluorescence detector and a surface detector array, independently with the Monte Carlo simulation [12].

In the TA FD analysis, the atmospheric density profile is obtained from radiosonde pressure and temperature from the Global Data Assimilation System (GDAS) database ${ }^{1}$. The typical atmospheric transparency is determined by LIDAR operation during the first two years operation [Q]. The absolute fluorescence yield is obtained from a measurement by Kakimoto et al. [एँ3], with a wavelength spectrum adopted from the result of the FLASH collaboration [14]]. The missing energy is corrected by a result from the CORSIKA [ए]] Monte Carlo simulation using the QGSJetII-03 model. In this paper, we address an impact on the energy scale due to the fluorescence yield parameters, and also study the missing energy evaluation using a technique of pioneered by the Pierre Auger Observatory.

\section{Fluorescence yield}

In TA FD analysis, we adopt the absolute fluorescence-photon yield measured by Kakimoto et al. [[13], and the relative intensity spectrum from FLASH measurements [[4]]. We call this "ModelK". The ELS result reported in ICRC 2015 [ए]] shows agreement within $2 \%$ to the fluorescence yield of the FLASH experiment [[4]] and "Model-A" which is the absolute yield measured with the AIRFLY experiment [155, [6] and the dependence on pressure, temperature and humidity is taken into account for each spectral line using the reference model formula reported in UHECR2012 [ㅍ]]. The ELS result shows a $26 \%$ higher result than Model-K and its uncertainty of ELS is quoted as $7.9 \%$ [ए]]. If the fluorescence yield measured by the ELS result is compared to Model-K, a systematic uncertainty is calculated by a quadratic sum of uncertainties on the ELS (7.9\%), detector calibration (10\%) and Model-K (10\%) [ए3]]. The total uncertainty is $16 \%$ thus that the ELS result shows $1.26 \pm 0.20$ compared to Model-K. If we consider a systematic uncertainty of the AIRFLY measurements (3.7\%) [प15], the ELS result indicates $1.02 \pm 0.14$ compared to Model-A.

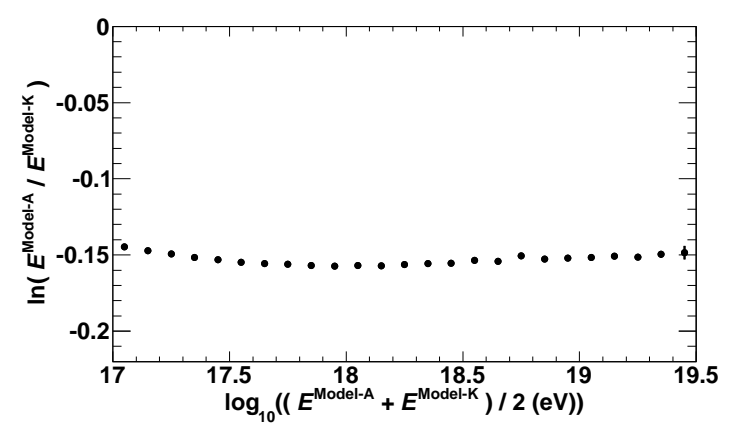

(a) Comparison to Model-A

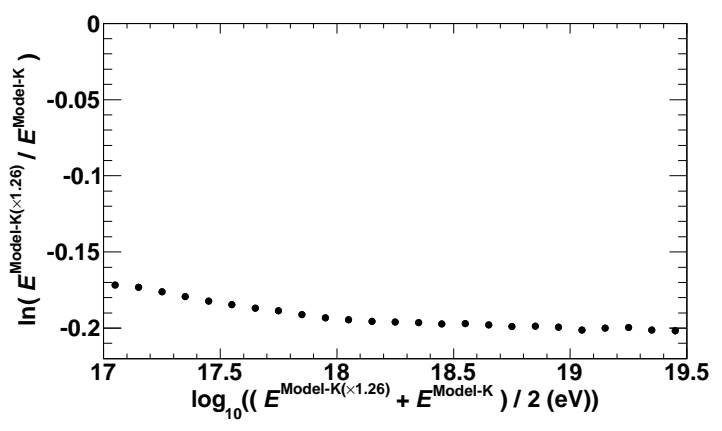

(b) Comparison to Model-K( $\times 1.26)$

Figure 2: An energy scale shift as a function of the primary energy if the Model-A (a) or Model- $\mathrm{K}(\times 1.26)$ (b) is assumed as fluorescence yield.

Here, we investigate an impact on the energy scale if we use different fluorescence yield models. The data is reanalyzed using Model-A and "Model- $\mathrm{K}(\times 1.26)$ " which is a 1.26 rescaling into

\footnotetext{
${ }^{1}$ http://ready.arl.noaa.gov/gdas1.php
} 
Model-K based on the ELS result. Model-K $(\times 1.26)$ shows consistent with a fluorescence yield of the FLASH [14] within 1\% [ए0]. Those reconstructed energies are compared to ones using Model-K. Figure $\square$ shows an energy scale shift as a function of primary energy if Model-A (a) or to Model- $\mathrm{K}(\times 1.26)(\mathrm{b})$ is assumed as fluorescence yield. Due to the Cherenkov dominated events in low energies, the energy shift is slightly smaller than one in high energies. The energy scale is changed by $-14 \%$ for Model-A and $-17 \%$ for Model-K $(\times 1.26)$.

A difference of the energy reduction between Model-A and Model-K $(\times 1.26)$ at high energies is caused by a difference of fluorescence spectral line. Figure B(a) shows an energy scale comparison between Model-A and Model-K( $\times 1.26)$. Figure B (b) shows a comparison of spectral line measured by several measurement in the laboratory [ए]]. A discrepancy around $380 \mathrm{~nm}$ between FLASH and AIRFLY spectral lines is affected in an attenuation traveling from an EAS axis to the FD station, introducing $2 \%$ difference at $10^{17} \mathrm{eV}$ and $5 \%$ difference at $10^{19} \mathrm{eV}$. Since the ELS is located at only $100 \mathrm{~m}$ away from FD station, it is impossible to investigate this spectral line discrepancy.

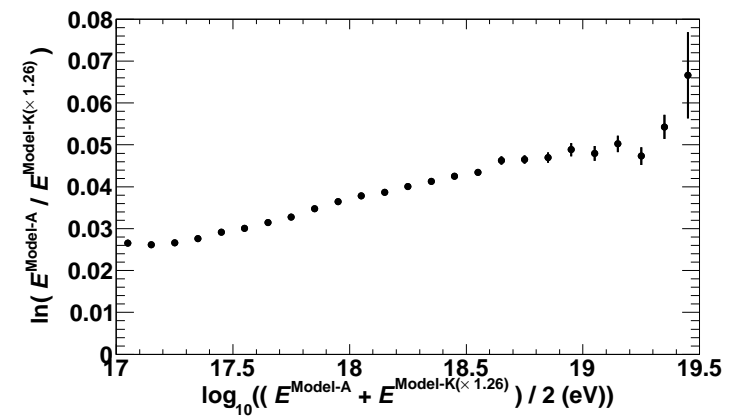

(a) Attenuation difference

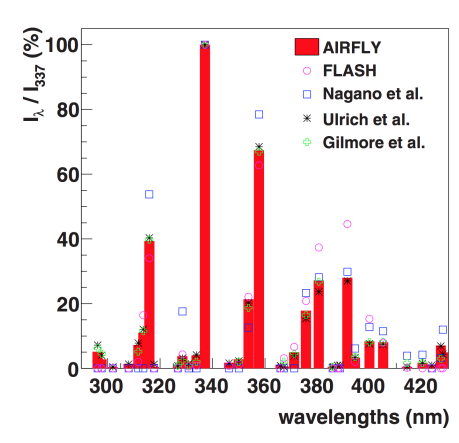

(b) Spectral line comparison

Figure 3: (a) A energy scale difference between Model-A and Model-K $(\times 1.26)$ as a function of primary energy. (b) The fluorescence line spectra among measurements in the laboratory taken from [ए]].

\section{Missing energy}

As pioneered in studies by the Pierre Auger Observatory [[2], we investigate a relationship between our observables in TA and the missing energy. The slant atmospheric depth from $X_{\max }$ (the depth in the atmosphere where the EAS reaches its maximum energy deposit) to the ground is defined as $D X=876.5 / \cos \theta-X_{\max }$, where $\theta$ is a zenith angle of EAS. Using the Monte Carlo simulation with TA FD and SD, the missing energy is parameterized by our observables, $D X$ and $S_{800}$ (the signal at $800 \mathrm{~m}$ from the core on the ground) by

$$
\log E_{\text {miss }}=A \log S_{800}+B
$$

in several ranges of $D X$. The obtained parameters of CORSIKA simulation using QGSJet01c are summarized in Table $\mathbb{W}$

Using this parameterization, the missing energy is estimated by the hybrid EAS events collected during 7 years. Figure $\$$ shows the missing energy as a function of a FD calorimetric energy, $E_{\text {cal }}$. This result is consistent with one from QGSJetII-03 proton above $10^{18.5} \mathrm{eV}$, while a $5 \%$ difference to one reported by Auger [미]. 


\begin{tabular}{c|c|c|c|c|c|c|c}
$D X\left(\mathrm{~g} / \mathrm{cm}^{2}\right)$ & $100-200$ & $200-300$ & $300-400$ & $400-500$ & $500-600$ & $600-700$ & $700-800$ \\
\hline$A$ & 1.059 & 1.053 & 1.086 & 1.085 & 1.045 & 1.117 & 1.142 \\
$B$ & 16.25 & 16.28 & 16.32 & 16.42 & 16.59 & 16.62 & 16.73
\end{tabular}

Table 1: Parameterizations of $A$ and $B$ in Equation 5.1 for several ranges of $D X$ to calculate the missing energy.

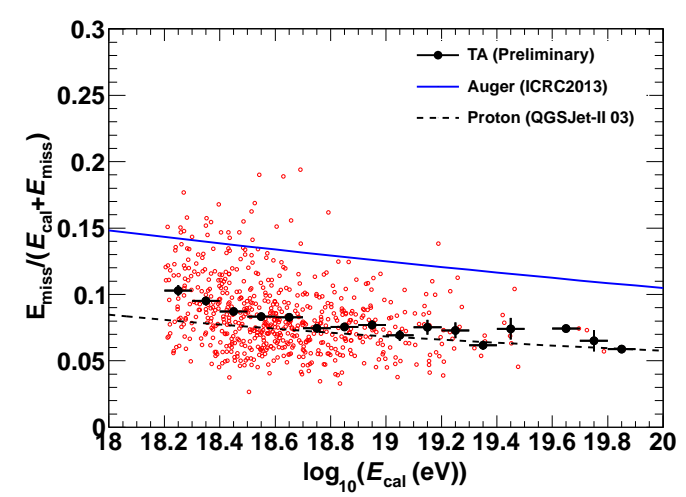

Figure 4: The missing energy (filled circle) calculated by the observables with TA FD and SD using the Parameterization of Table 1 as a function of calorimetric energy measured with FD. As a reference, the missing energy obtained by QGSJetII-03 proton and one reported by Auger [2] are indicated as dashed line and solid line, respectively.

\section{Conclusions}

We studied an systematic uncertainty on the energy scale with the Telescope Array fluorescence detectors. If different models of the fluorescence yield are adopted in the FD analysis, the energy scale is changed by $-14 \%$ for Model-A and $-17 \%$ for Model- $\mathrm{K}(\times 1.26)$ compared to the current model, Model-K. The missing energy is evaluated from observed data collected by the TA FD and TA SD using a technique pioneered by the Pierre Auger Observatory. The result is consistent with one from QGSJetII-03 proton above $10^{18.5} \mathrm{eV}$, while a $5 \%$ difference to one reported by Auger.

\section{Acknowledgements}

The Telescope Array experiment is supported by the Japan Society for the Promotion of Science through Grants-in-Aid for Scientific Research on Specially Promoted Research (21000002) "Extreme Phenomena in the Universe Explored by Highest Energy Cosmic Rays" and for Scientific Research (19104006), and the Inter-University Research Program of the Institute for Cosmic Ray Research; by the U.S. National Science Foundation awards PHY-0307098, PHY-0601915, PHY-0649681, PHY-0703893, PHY-0758342, PHY-0848320, PHY-1069280, PHY-1069286, PHY1404495 and PHY-1404502; by the National Research Foundation of Korea (2007-0093860, R3210130, 2012R1A1A2008381, 2013004883); by the Russian Academy of Sciences, RFBR grants 11-02-01528a and 13-02-01311a (INR), IISN project No. 4.4502.13, and Belgian Science Policy under IUAP VII/37 (ULB). The foundations of Dr. Ezekiel R. and Edna Wattis Dumke, Willard L. 
Eccles, and George S. and Dolores Doré Eccles all helped with generous donations. The State of Utah supported the project through its Economic Development Board, and the University of Utah through the Office of the Vice President for Research. The experimental site became available through the cooperation of the Utah School and Institutional Trust Lands Administration (SITLA), U.S. Bureau of Land Management, and the U.S. Air Force. We also wish to thank the people and the officials of Millard County, Utah for their steadfast and warm support. We gratefully acknowledge the contributions from the technical staffs of our home institutions. An allocation of computer time from the Center for High Performance Computing at the University of Utah is gratefully acknowledged.

\section{References}

[1] Telescope Array Collaboration, M. Fukushima et al., Telescope array project for extremely high energy cosmic rays, Prog.Theor.Phys.Suppl. 151 (2003) 206-210.

[2] Telescope Array Collaboration, T. Abu-Zayyad et al., The surface detector array of the Telescope Array experiment, Nucl.Instrum.Meth. A689 (2012) 87-97,

[3] H. Tokuno, Y. Tameda, M. Takeda, K. Kadota, D. Ikeda, et al., New air fluorescence detectors employed in the Telescope Array experiment, Nucl.Instrum.Meth. A676 (2012) 54-65,

[4] HiRes Collaboration, R. Abbasi et al., First observation of the Greisen-Zatsepin-Kuzmin suppression, Phys.Rev.Lett. 100 (2008) 101101, [astro-ph/0703099].

[5] S. Kawana, N. Sakurai, T. Fujii, M. Fukushima, N. Inoue, et al., Calibration of Photomultiplier Tubes for the Fluorescence Detector of Telescope Array Experiment using a Rayleigh Scattered Laser Beam, Nucl.Instrum.Meth. A681 (2012) 68-77, [1202.1934].

[6] H. Tokuno, Y. Murano, S. Kawana, Y. Tameda, A. Taketa, et al., On site calibration for new fluorescence detectors of the telescope array experiment, Nucl.Instrum.Meth. A601 (2009) 364-371.

[7] B. K. Shin, H. Tokuno, Y. Tsunesada, T. Abu-Zayyad, R. Aida, et al., Gain monitoring of telescope array photomultiplier cameras for the first 4 years of operation, Nucl.Instrum.Meth. A768 (2014) 96-103.

[8] S. Udo, R. Cady, M. Fukushima, J. Matthews, T. Jason, et al., The Central Laser Facility at the Telescope Array, Proc. of the 30th International Cosmic Ray Conference, Merida, Mexico 5 (2007) 1021-1024.

[9] T. Tomida, Y. Tsuyuguchi, T. Arai, T. Benno, M. Chikawa, et al., The atmospheric transparency measured with a LIDAR system at the Telescope Array experiment, Nucl.Instrum.Meth. $\mathbf{A 6 5 4}$ (2011) 653-660,

[10] B. Shin, Calibration of the TA Fluorescence Detectors with Electron Light Source, PoS ICRC2015 (2016) 640 .

[11] D. Heck, G. Schatz, T. Thouw, J. Knapp, and J. Capdevielle, CORSIKA: A Monte Carlo code to simulate extensive air showers, Forschungszentrum Karlsruhe Report FZKA (1998) 6019.

[12] Pierre Auger Collaboration, A. Aab et al., The Pierre Auger Observatory: Contributions to the 33rd International Cosmic Ray Conference, ICRC 2013 (2013)

[13] F. Kakimoto, E. Loh, M. Nagano, H. Okuno, M. Teshima, et al., A Measurement of the air fluorescence yield, Nucl.Instrum.Meth. A372 (1996) 527-533. 
[14] R. Abbasi, T. Abu-Zayyad, K. Belov, J. Belz, Z. Cao, et al., Air fluorescence measurements in the spectral range 300-420 nm using a 28.5-GeV electron beam, Astropart.Phys. 29 (2008) 77-86, $[0708.3116]$.

[15] AIRFLY Collaboration, M. Ave et al., Precise measurement of the absolute fluorescence yield of the $337 \mathrm{~nm}$ band in atmospheric gases, Astropart.Phys. 42 (2013) 90-102, [1210.6734]].

[16] AIRFLY Collaboration, M. Ave et al., Measurement of the pressure dependence of air fluorescence emission induced by electrons, Astropart.Phys. 28 (2007) 41-57, [astro-ph/0703132].

[17] B. Keilhauer, M. Bohacova, M. Fraga, J. Matthews, N. Sakaki, Y. Tameda, Y. Tsunesada, and A. Ulrich, Nitrogen fluorescence in air for observing extensive air showers, EPJ Web Conf. 53 (2013) 01010, 